\title{
Giant Colonic Diverticulum: Diagnosis on CT Scan
}

\section{Hamza Wani*, Sadananda Meher, Pramod Kumar Samantaray and Mohammad Ibrarullah}

Department of Surgical Gastroenterology, Apollo Hospitals, Bhubaneshwar, India

*Corresponding Author: Hamza Wani, Department of Surgical Gastroenterology, Apollo Hospitals, Bhubaneshwar, India.

Received: July 03, 2019; Published: July 23, 2019

DOI: $10.31080 /$ ASGIS.2019.02.0063

\begin{abstract}
We report CT scan images of a giant right colonic diverticulum presenting as a right sided abdominal lump in a 78 year old female.

Keywords: Giant Diverticulum; Abdominal Lump; Diverticulosis; Constipation
\end{abstract}

A 78-year-old-female presented with chronic constipation and colicky abdominal pain for six months. Abdominal examination revealed a smooth, non tender lump in the right lumbar region approximately ten $\mathrm{cm}$ in diameter. On contrast CT scan, a heterogenous mass $(10 \mathrm{~cm} \times 10 \mathrm{~cm})$ with air pockets, was observed in relation to ascending colon (Figure $1 \mathrm{a}, \mathrm{b}$ ). Colonoscopy revealed multiple diverticula all along the colon and a wide mouth diverticulum at the junction of caecum and ascending colon (Figure 2a) containing inspissated fecal matter. At laparotomy, the mass was confirmed to be a giant diverticulum arising from ascending colon, which was subsequently excised (Figure $2 b$ ).

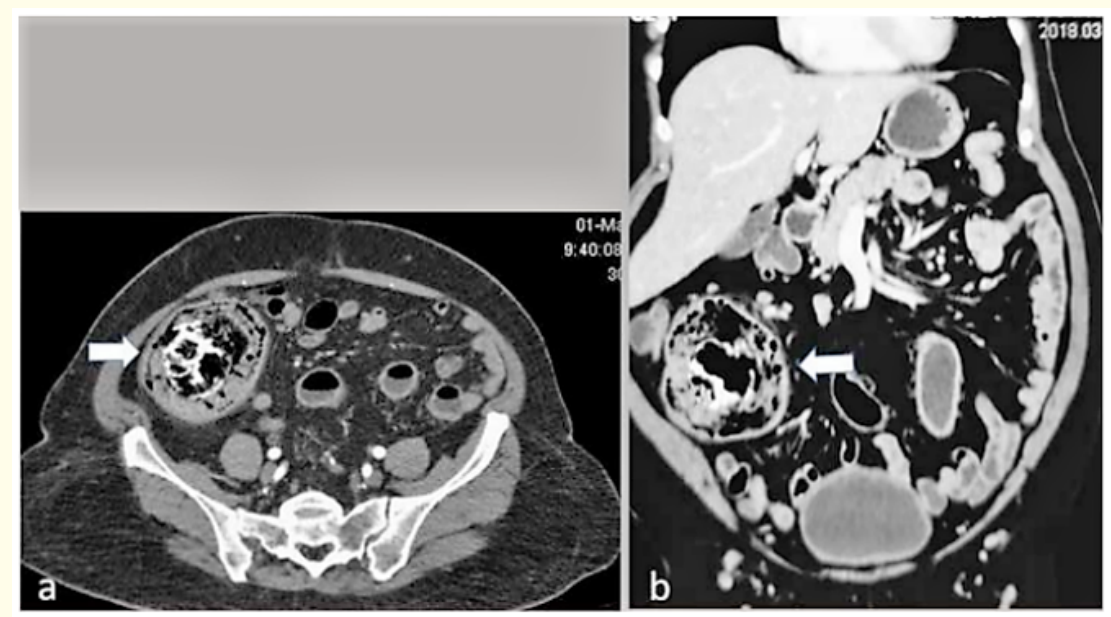

Figure 1: (a). CECT abdomen showing a heterogenous large mass (arrow) in relation to caecum and proximal ascending colon. (b) coronal reconstruction of the same. 

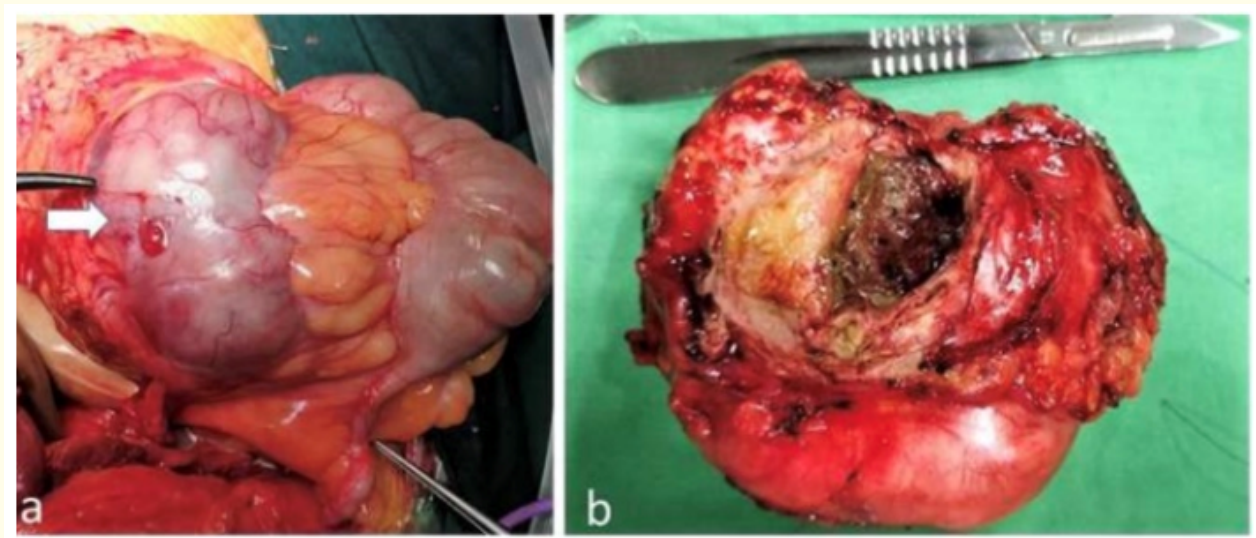

Figure 2: (a) Operative finding of a giant colonic diverticulum (arrow) at the junction of caecum and ascending colon. (b) Excised diverticulum with inspissated fecal matter inside.

A giant colonic diverticulum (GCD) is defined as a large diverticulum more than $4 \mathrm{~cm}$ in diameter. Through a secondary ball valve mechanism, gas enters the diverticulum but is unable to leave, leading to the formation of a GCD. About $33-48 \%$ of giant diverticula present as palpable lump \& mostly in relation to sigmoid colon [1,2]. Giant diverticulum arising from right colon is extremely rare, particularly in India, where the disease itself is uncommon. Excision is usually recommended to prevent complications.

\section{Bibliography}

1. Steenvoorde P and Vogelaar FJ. "Giant colonic diverticula, review of diagnostic and therapeutic options". Digestive Surgery 21 (2004): 1-6.

2. Nigri G and Petrucciani N. "Giant colonic diverticulum: Clinical presentation, diagnosis and treatment: Systematic review of 166 cases". World Journal of Gastroenterology 21.1 (2015): 360-368.

\section{Volume 2 Issue 6 August 2019}

(C) All rights are reserved by Hamza Wani., et al. 